(1)

CrossMark

\title{
Outcomes in coronary artery disease patients with sleepy obstructive sleep apnoea on CPAP
}

\author{
Yüksel Peker (10), ${ }^{1,2}$, Erik Thunström (10) ${ }^{2,3}$, Helena Glantz $\mathbb{1}^{4}$, Karl Wegscheider $\mathbb{( i D}^{5}$ \\ and Christine Eulenburg $\mathbb{1}^{6}$
}

\begin{abstract}
Affiliations: ${ }^{1}$ Dept of Pulmonary Medicine, Faculty of Medicine, Marmara University, Istanbul, Turkey. ${ }^{2}$ Dept of Molecular and Clinical Medicine/Cardiology, Sahlgrenska Academy, University of Gothenburg, Gothenburg, Sweden. ${ }^{3}$ Dept of Cardiology, Sahlgrenska University Hospital/Östra, Gothenburg, Sweden. ${ }^{4}$ Dept of Internal Medicine, Skaraborg Hospital, Lidköping. Sweden. ${ }^{5}$ Dept of Medical Biometry and Epidemiology, University Medical Center Hamburg-Eppendorf, Hamburg, Germany. ${ }^{6}$ Medical Statistics and Decision Making, Dept of Epidemiology, University Medical Center Groningen, Groningen, The Netherlands.
\end{abstract}

Correspondence: Yüksel Peker, Dept of Pulmonary Medicine, Faculty of Medicine, Marmara University, Pendik Education and Research Hospital, Sleep Medicine Center, 34899 Pendik, Istanbul, Turkey. E-mail: yuksel.pekeramarmara.edu.tr

\section{@ERSpublications}

Cardiovascular risk in coronary artery disease patients with OSA on CPAP treatment is similar to those without OSA http://ow.ly/hB1z30eXxFx

Cite this article as: Peker Y, Thunström E, Glantz H, et al. Outcomes in coronary artery disease patients with sleepy obstructive sleep apnoea on CPAP. Eur Respir J 2017; 50: 1700749 [https://doi.org/10.1183/ 13993003.00749-2017].

ABSTRACT Coronary artery disease (CAD) patients with obstructive sleep apnoea (OSA) have increased risk for major adverse cardiovascular and cerebrovascular events (MACCEs) compared with CAD patients without OSA. We aimed to address if the risk is similar in both groups when OSA patients are treated.

This study was a parallel observational arm of the RICCADSA randomised controlled trial, conducted in Sweden between 2005 and 2013. Patients with revascularised CAD and OSA (apnoea-hypopnoea index $(\mathrm{AHI}) \geqslant 15$ events $\cdot \mathrm{h}^{-1}$ ) with daytime sleepiness (Epworth Sleepiness Scale score $\geqslant 10$ ) were offered continuous positive airway pressure (CPAP) $(\mathrm{n}=155)$; CAD patients with no OSA (AHI $<5$ events. $\mathrm{h}^{-1}$ ) acted as controls $(n=112)$, as a randomisation of sleepy OSA patients to no treatment would not be ethically feasible. The primary end-point was the first event of MACCEs. Median follow-up was 57 months.

The incidence of MACCEs was $23.2 \%$ in OSA patients versus $16.1 \%$ in those with no OSA (adjusted hazard ratio $0.96,95 \%$ CI $0.40-2.31$; $\mathrm{p}=0.923$ ). Age and previous revascularisation were associated with increased risk for MACCEs, whereas coronary artery bypass grafting at baseline was associated with reduced risk.

We conclude that the risk for MACCEs was not increased in CAD patients with sleepy OSA on CPAP compared with patients without OSA.

This article has supplementary material available from erj.ersjournals.com

Received: April 102017 | Accepted after revision: Aug 312017

Support statement: This study was supported by grants from the Swedish Research Council; the Swedish Heart-Lung Foundation, the "Agreement concerning research and education of doctors" of Västra Götalandsregionen (ALFGBG), research fund at Skaraborg Hospital, Skaraborg Research and Development Council, the Heart Foundation of Kärnsjukhuset, and ResMed Foundation and ResMed Ltd. ResMed Sweden provided some of the sleep recording devices and technical support. None of the funders had any direct influence on the design of the study, analysis of the data, data collection, drafting of the manuscript or decision to publish. Funding information for this article has been deposited with the Crossref Funder Registry.

Conflict of interest: Disclosures can be found alongside this article at erj.ersjournals.com

Copyright OERS 2017. This ERJ Open article is open access and distributed under the terms of the Creative Commons Attribution Licence 4.0. 


\section{Introduction}

Nearly half of clinical populations with coronary artery disease (CAD) also have obstructive sleep apnoea (OSA) and these individuals have worse prognosis compared with CAD patients without OSA [1]. Moreover, despite advances in medical treatment and revascularisation techniques, many CAD patients undergoing percutaneous coronary intervention (PCI) or coronary artery bypass grafting (CABG) experience major adverse cardiac and cerebrovascular events (MACCEs) in the years following the intervention [2,3]. It has been suggested that OSA may contribute to the occurrence of MACCEs in revascularised CAD cohorts. In a 6-month follow-up study after PCI, MACCEs were observed in almost $24 \%$ of patients with concomitant OSA compared with $5 \%$ of those without OSA [4]. More recent data support an independent relationship between OSA and subsequent MACCEs in patients undergoing PCI $[5,6]$, as well as in those treated with CABG [7].

Continuous positive airway pressure (CPAP) is recommended as first-line treatment for OSA, and is associated with reduced daytime sleepiness and improved quality of life in symptomatic patients [8]. However, the majority of CAD patients with OSA do not experience daytime sleepiness and there is currently no clearly established rationale for treatment in such patients. CPAP has been shown to be beneficial in patients with CAD and OSA who are adherent to treatment [9-11]. An observational, nonrandomised study suggested that patients who received CPAP treatment for OSA had reduced cardiac mortality at 5 years after PCI compared with those who declined CPAP treatment [12]. Until recently, there was a lack of long-term prospective randomised controlled trials (RCTs) to address whether cardiac patients with nonsleepy OSA should be offered CPAP treatment to reduce MACCEs.

The RICCADSA (Randomised Intervention with CPAP in CAD and OSA) trial was the first RCT to investigate the impact of CPAP on MACCEs in revascularised CAD patients and nonsleepy OSA [13]. The results showed that routine CPAP prescription to CAD patients with nonsleepy OSA did not significantly reduce long-term adverse outcomes in the intention-to-treat (ITT) population. A reduction in risk for adverse events was only observed after adjustment for baseline comorbidities and CPAP adherence [13]. In a recent report from an RCT conducted in a much larger cohort of patients with moderate-to-severe OSA and established cardiovascular disease, CPAP treatment plus usual care did not prevent cardiovascular events in the ITT population compared with usual care alone, despite a significant reduction in daytime sleepiness and improvement in health-related quality of life and mood [14].

This analysis was conducted in the observational arm of the RICCADSA cohort and investigated the risk for MACCEs in CAD patients with sleepy OSA treated with CPAP compared with CAD patients who did not have OSA, as a randomisation of untreated sleepy OSA patients to control would not be ethically feasible. The impact of CPAP on daytime sleepiness in patients with sleepy OSA was also evaluated.

\section{Material and methods}

\section{Study subjects}

The RICCADSA study methodology has been published previously [15] and is detailed in the supplementary material. In summary, the study population consisted of adult patients with angiography-verified CAD who had undergone PCI or CABG in Skaraborg County, Sweden, in the previous 6 months and had an apnoea-hypopnoea index (AHI) of $<5$ or $\geqslant 15$ events $\cdot \mathrm{h}^{-1}$ during a sleep study. Patients with borderline OSA (AHI 5.0-14.9 events. $\mathrm{h}^{-1}$ ) and those with predominant $(>50 \%)$ central apnoeas and hypopnoeas of Cheyne-Stokes nature were excluded (figure 1). Patients were recruited between December 2005 and November 2010, and follow-up was completed in May 2013 with a predefined minimum follow-up of 2 years. CAD patients with nonsleepy OSA (AHI $\geqslant 15$ events $\cdot \mathrm{h}^{-1}$, Epworth Sleepiness Scale (ESS) score <10) were included in the RCT arm [13]. Patients with a sleepy OSA phenotype (AHI $\geqslant 15$ events $\mathrm{h}^{-1}$, ESS score $\geqslant 10$ ) who were receiving CPAP and CAD patients without OSA were included in the observational arm and followed prospectively (figure 1).

The RICCADSA study protocol was approved by the Ethics Committee of the Medical Faculty of the University of Gothenburg, Gothenburg, Sweden (approval 207-05: September 13, 2005; amendment T744-10: November 26, 2010; amendment T512-11: June 16, 2011) and all patients provided written informed consent. The trial was registered with the national researchweb.org (FoU i Sverige (Research and Development in Sweden): identifier VGSKAS-4731; April 29, 2005) and with ClinicalTrials.gov (identifier NCT00519597).

\section{Study design}

A blinded interim analysis was conducted in February 2010 and the protocol was amended with a new power calculation for the primary end-points of the RCT arm [13]. Based on the interim analysis of group assignment and the inclusion rate in February 2010, approximately 150 patients with sleepy OSA and 110 patients without OSA were expected to be included in the observational arm during the inclusion of the 
RCT arm. There was only one report [4] with a cohort of 89 patients with revascularised CAD (51 OSA versus 38 no OSA) available at the time of the interim analysis; therefore, the expected number of patients included in the observational arm $(n=260)$ was considered to be sufficient to address the research question.

An independent clinical event committee, unaware of patient identities and group allocation, reviewed all data obtained from hospital records and death certificates by the end of May 2013. Moreover, a random $10 \%$ selection of the database for baseline clinical data and follow-up procedures, including CPAP adherence and primary end-points, was monitored by a data monitoring board.

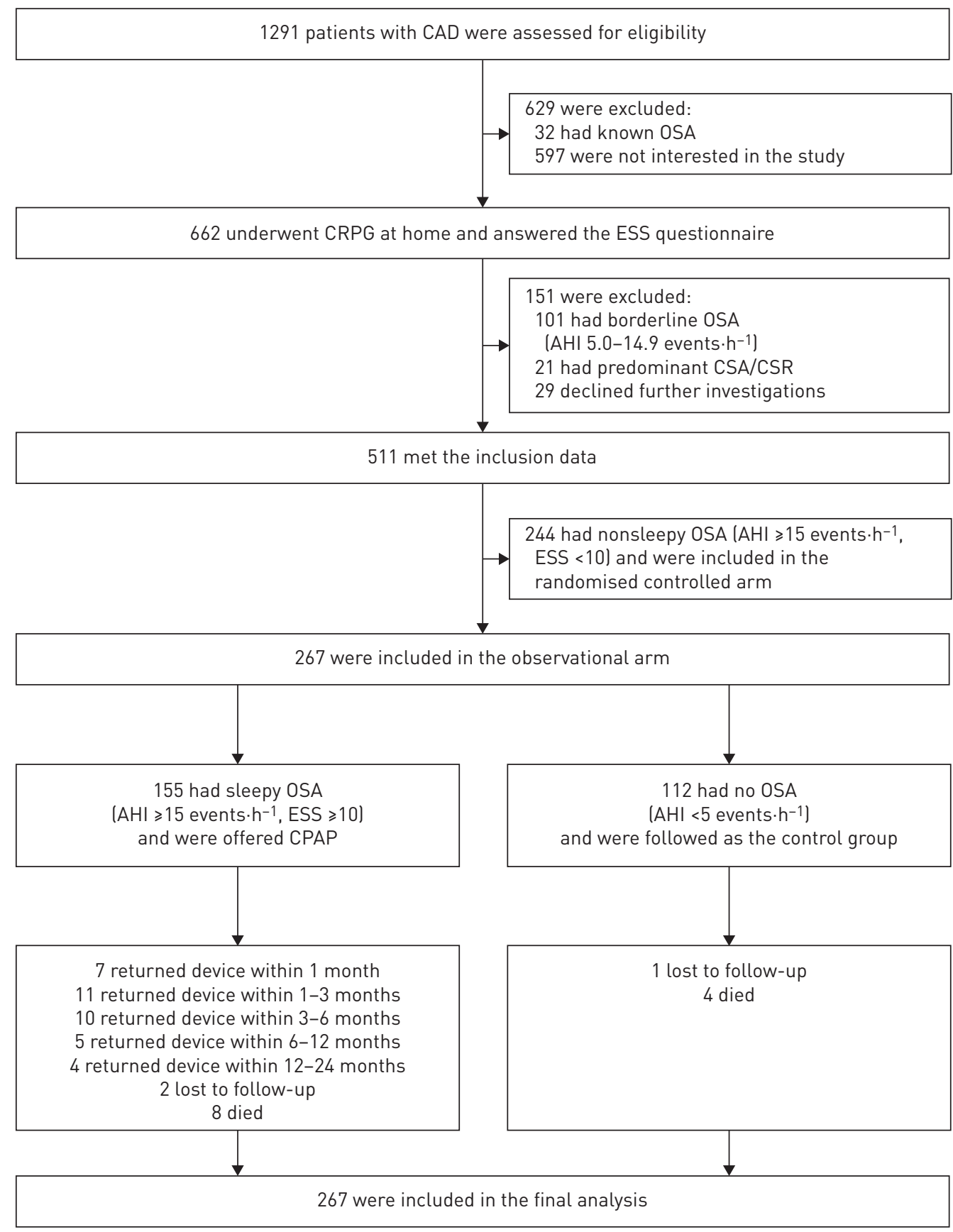

FIGURE 1 Flow of patients through the study. CAD: coronary artery disease; OSA: obstructive sleep apnoea; CRPG: cardiorespiratory polygraphy; ESS: Epworth Sleepiness Scale; AHI: apnoea-hypopnoea index; CSA: central sleep apnoea; CSR: Cheyne-Stokes respiration; CPAP: continuous positive airway pressure. 


\section{Methods}

Full details of home sleep recordings (cardiorespiratory polygraphy (CRPG)) and in-hospital polysomnography (PSG) for the OSA group are provided in the supplementary material. Sleepy OSA patients were offered CPAP treatment and fitted with an automatic CPAP device (S8 or S9; ResMed, San Diego, CA, USA) by trained staff. Additional follow-up details, including adherence to CPAP treatment, are provided in the supplementary material.

\section{Outcomes}

The primary end-point was the rate of MACCEs (repeat revascularisation, myocardial infarction, stroke and cardiovascular mortality). Data were obtained from patients' medical records and, when necessary, from the Swedish Hospital Discharge Register as well as the Swedish National Cause of Death Registry. As described previously, each event was evaluated separately and as part of the combined end-point [13]. For patients who experienced more than one event during the follow-up period, only the first event was included in the combined end-point. Secondary end-points included all-cause mortality and acute hospital admission for cardiovascular reasons. Cardiovascular diagnosis criteria defined by the independent clinical event committee are available in the supplementary material. The clinical indication for CPAP prescription to CAD patients with sleepy OSA was mainly to reduce daytime sleepiness and therefore ESS scores were evaluated separately.

\section{Analysis}

Descriptive statistics are given as mean and standard deviation or number (percentage). For baseline differences between the groups, the Chi-squared test and t-test were applied. Total sleep time, time spent in the supine position and AHI values from repeated sleep recordings (CRPG versus PSG) at the individual level, as well as changes in ESS score and body mass index (BMI) from baseline over time within the OSA group, were compared with the paired t-test. Pearson correlation analysis was performed to test the linear relationship between AHI values from CRPG versus PSG.

Kaplan-Meier analyses and Cox proportional hazards models were used to estimate the impact of OSA on the primary end-point. Multivariate adjustment was made for age, sex, baseline AHI, BMI, current smoking, revascularisation type, former revascularisation, acute myocardial infarction, hypertension, diabetes mellitus, lung disease and left ventricular ejection fraction (LVEF) at baseline. A secondary analysis was performed after excluding patients who died or were lost to follow-up, or returned the CPAP device before the predefined minimum follow-up of 2 years. For the on-treatment analysis of the OSA group, a time-dependent Cox model [16] was used to estimate the impact of CPAP usage (cut-off levels of $4 \mathrm{~h}$ per night) on the primary end-point. As described previously in the RCT arm of the RICCADSA trial [12], this approach accounts for the time-varying character of the intervention because subject follow-up is split into multiple intervals according to the visit dates of the CPAP usage evaluation. Visits were planned after 1, 3, 6 and 12 months, and then annually until the end of the study. Incomplete usage data due to missed visits were replaced. One missing episode was replaced by the last observation, if the missing episode was followed by a visit. Two or more subsequent missing visits were replaced by 0 . If the data from the first visit after 1 month were missing, they were replaced by the usage data of the 3-month visit. For the on-treatment analysis of the OSA group, multivariate adjustment was made for CPAP nights/ period, and age, sex, baseline AHI, BMI, current smoking, revascularisation type, former revascularisation, acute myocardial infarction, hypertension, diabetes mellitus, lung disease and LVEF at baseline.

All statistical tests were two-sided and $\mathrm{p}<0.05$ was considered statistically significant. Statistical analysis was performed using SPSS version 22.0 for Windows (IBM, Armonk, NY, USA) and Stata version 14 (StataCorp, College Station, TX, USA).

\section{Results}

\section{Study participants}

A consecutive population of 1259 patients met the inclusion criteria for screening, of whom 662 (52.7\%) agreed to participate in the sleep study (figure 1). Diagnostic CRPG was performed at home an average of 63 days (median (interquartile range (IQR)) 59 (42-78) days) after mechanical revascularisation and patients fulfilling the inclusion criteria underwent baseline investigations on average 35 days (median (IQR) 30 (20-45) days) after home sleep recordings.

\section{Baseline characteristics}

A total of 267 patients with CAD and OSA fulfilled the inclusion criteria for the observational arm. As shown in table 1, sleepy OSA patients were more likely to be male, and had a higher rate of obesity, diabetes mellitus and three-vessel disease compared with CAD patients without OSA. Intervention with CABG at baseline and use of calcium channel blockers tended to be more common in the OSA group, and sleepy 
TABLE 1 Demographic and clinical characteristics of study patients at baseline

\begin{tabular}{|c|c|c|c|}
\hline & Sleepy OSA & No OSA & p-value \\
\hline Subjects $\mathrm{n}$ & 155 & 112 & \\
\hline Age years & $62.6 \pm 7.3$ & $61.5 \pm 9.6$ & 0.287 \\
\hline $\mathrm{AHI}$ events $\cdot \mathrm{h}^{-1}$ & $32.1 \pm 16.2$ & $3.0 \pm 1.3$ & $<0.001$ \\
\hline ODI events $\cdot \mathrm{h}^{-1}$ & $21.0 \pm 16.6$ & $1.6 \pm 1.3$ & $<0.001$ \\
\hline ESS score & $12.2 \pm 2.6$ & $5.7 \pm 2.9$ & $<0.001$ \\
\hline BMI $\mathrm{kg} \cdot \mathrm{m}^{-2}$ & $29.7 \pm 4.4$ & $25.6 \pm 3.0$ & $<0.001$ \\
\hline LVEF \% & $57.7 \pm 8.2$ & $58.2 \pm 7.3$ & 0.479 \\
\hline Obesity & 41.3 & 7.1 & $<0.001$ \\
\hline Female & 11.0 & 25.9 & 0.003 \\
\hline Current smoker & 17.4 & 25.9 & 0.093 \\
\hline Pulmonary disease & 9.7 & 13.4 & 0.343 \\
\hline Hypertension & 57.4 & 46.2 & 0.076 \\
\hline Acute MI at baseline & 49.0 & 58.9 & 0.110 \\
\hline Three-vessel disease & 21.6 & 10.9 & 0.024 \\
\hline CABG at baseline & 25.8 & 16.1 & 0.057 \\
\hline Previous $\mathrm{PCI}$ or $\mathrm{CABG}$ & 20.0 & 16.1 & 0.413 \\
\hline Diabetes mellitus & 25.2 & 13.4 & 0.018 \\
\hline$\beta$-Blocker use & 91.4 & 79.6 & 0.006 \\
\hline Diuretic use & 21.7 & 9.3 & 0.008 \\
\hline CCB use & 21.1 & 12.0 & 0.058 \\
\hline ACE inhibitor use & 37.5 & 39.8 & 0.705 \\
\hline ARB use & 15.8 & 6.5 & 0.022 \\
\hline Anticoagulant use $\mathrm{e}^{\#}$ & 99.4 & 100.0 & 0.401 \\
\hline Lipid-lowering agent use & 95.4 & 90.7 & 0.126 \\
\hline
\end{tabular}

Data are presented as mean \pm SD or $\%$, unless otherwise stated. OSA: obstructive sleep apnoea; AHI: apnoea-hypopnoea index; ODI: oxygen desaturation index; ESS: Epworth Sleepiness Scale; BMI: body mass index; LVEF: left ventricular ejection fraction; MI: myocardial infarction; CABG: coronary artery bypass grafting; $\mathrm{PCl}$ : percutaneous coronary intervention; $\mathrm{CCB}$ : calcium channel blocker; ACE: angiotensin converting enzyme; ARB: angiotensin II receptor blocker. " : aspirin and/or clopidogrel and/or warfarin.

OSA patients were significantly more likely to be using a $\beta$-blocker, diuretics and an angiotensin receptor blocker (table 1). The number and type of stents, treated lesions, intervention type (acute/subacute or elective), and type of CABG grafts did not differ significantly between the groups (supplementary table E1).

\section{Numbers analysed}

Median (IQR) follow-up time until death, loss to follow-up or the end of the study was 57.1 (43.772.9) months. All patients were included in the final analysis for primary outcomes; 12 patients died and three were lost to follow-up (figure 1). Of 155 patients with AHI $\geqslant 15$ events $\cdot h^{-1}$ on CRPG, one had AHI $<5$ events $\cdot h^{-1}$ on in-hospital PSG the day before the initiation of CPAP (60 days after initial at-home CRPG). Follow-up data of this patient and correlations between AHI values on CRPG versus PSG in OSA patients are provided in supplementary table E2.

Of the 155 OSA patients who started CPAP at baseline, two died, one was lost to follow-up, 37 of the remaining $152(24.3 \%)$ returned the device within 2 years and $71.8 \%$ were on CPAP until the first event or the end of the study. OSA patients who returned the device within 2 years had less severe OSA in terms of AHI and oxygen desaturation index, were less obese, less likely to have had acute myocardial infarction at baseline, and less likely to be using diuretics compared with patients remaining on CPAP at 2-year follow-up (table 2). CPAP compliance based on CPAP device data is shown in supplementary table E3, demonstrating an increasing proportion of patients returning the device over time but also increasing CPAP compliance (hours per night) among patients remaining on treatment. In the OSA group, BMI increased significantly from $29.9 \pm 4.3 \mathrm{~kg} \cdot \mathrm{m}^{-2}$ at baseline to $30.2 \pm 4.3 \mathrm{~kg} \cdot \mathrm{m}^{-2}$ at 3 -month follow-up, to 30.9 $\pm 4.7 \mathrm{~kg} \cdot \mathrm{m}^{-2}$ at 1 -year follow-up and to $31.0 \pm 5.1 \mathrm{~kg} \cdot \mathrm{m}^{-2}$ at 2 -year follow-up (all $\mathrm{p}<0.001$ versus baseline). In the no OSA group, there was also a significant increase in BMI, from $25.5 \pm 3.2 \mathrm{~kg} \cdot \mathrm{m}^{-2}$ at baseline to $25.9 \pm 3.4 \mathrm{~kg} \cdot \mathrm{m}^{-2}$ at 1 -year follow-up $(\mathrm{p}=0.040)$ and to $26.3 \pm 3.2 \mathrm{~kg} \cdot \mathrm{m}^{-2}$ at 2 -year follow-up $(\mathrm{p}<0.001)$.

\section{Outcomes}

Overall, 54 patients reached the combined end-point during follow-up: 36 (23.2\%) in the CPAP-treated sleepy OSA group and $18(16.1 \%)$ in the no OSA group $(\mathrm{p}=0.151)$. The incidence of the composite 
TABLE 2 Demographic and clinical characteristics of the obstructive sleep apnoea patients adherent and nonadherent to continuous positive airway pressure at the predefined minimum follow-up of 2 years ${ }^{\#}$

\begin{tabular}{|c|c|c|c|}
\hline & Nonadherent & Adherent & p-value \\
\hline Subjects $n$ & 37 & 115 & \\
\hline Age years & $63.7 \pm 7.8$ & $62.4 \pm 7.2$ & 0.338 \\
\hline$A H I$ events $\cdot h^{-1}$ & $28.6 \pm 11.8$ & $33.5 \pm 17.3$ & 0.055 \\
\hline ODI events $\cdot h^{-1}$ & $17.3 \pm 10.7$ & $22.6 \pm 10.8$ & 0.043 \\
\hline ESS score & $12.2 \pm 2.0$ & $12.1 \pm 2.5$ & 0.775 \\
\hline BMI $\mathrm{kg} \cdot \mathrm{m}^{-2}$ & $28.7 \pm 3.2$ & $30.1 \pm 4.7$ & 0.043 \\
\hline LVEF \% & $56.5 \pm 9.2$ & $58.0 \pm 7.9$ & 0.350 \\
\hline Obesity & 37.8 & 42.6 & 0.608 \\
\hline Female & 10.8 & 11.3 & 0.601 \\
\hline Current smoker & 18.9 & 15.7 & 0.641 \\
\hline Pulmonary disease & 13.5 & 7.8 & 0.298 \\
\hline Hypertension & 59.5 & 57.4 & 0.825 \\
\hline Acute $\mathrm{MI}$ at baseline & 27.0 & 55.7 & 0.002 \\
\hline Three-vessel disease & 25.7 & 20.0 & 0.473 \\
\hline CABG at baseline & 32.4 & 23.5 & 0.278 \\
\hline Previous $\mathrm{PCl}$ or CABG & 24.3 & 19.1 & 0.495 \\
\hline Diabetes mellitus & 24.3 & 24.3 & 0.998 \\
\hline$\beta$-Blocker use & 88.9 & 90.4 & 0.787 \\
\hline Diuretic use & 11.1 & 27.8 & 0.045 \\
\hline CCB use & 19.4 & 26.1 & 0.419 \\
\hline ACE inhibitor use & 36.1 & 39.1 & 0.745 \\
\hline ARB use & 19.4 & 17.4 & 0.779 \\
\hline Aspirin use & 86.1 & 88.7 & 0.676 \\
\hline Clopidogrel use & 69.4 & 53.9 & 0.100 \\
\hline Warfarin use & 8.3 & 11.3 & 0.763 \\
\hline Lipid-lowering agent use & 91.7 & 93.9 & 0.636 \\
\hline
\end{tabular}

Data are presented as mean \pm SD or \%, unless otherwise stated. AHI: apnoea-hypopnoea index; ODI: oxygen desaturation index; ESS: Epworth Sleepiness Scale; BMI: body mass index; LVEF: left ventricular ejection fraction; $\mathrm{MI}$ : myocardial infarction; CABG: coronary artery bypass grafting; $\mathrm{PCl}$ : percutaneous coronary intervention; CCB: calcium channel blocker; ACE: angiotensin converting enzyme; ARB: angiotensin II receptor blocker. \#: three patients from the entire cohort (two patients who died and one who was lost to follow-up before the 2-year follow-upl were excluded.

end-point was 4.72 (95\% CI 3.61-6.16) per 100 person-years in the whole cohort; 5.57 (95\% CI 4.02-7.73) per 100 person-years in the OSA group on CPAP versus 3.61 (95\% CI 2.27-5.73) per 100 person-years in no OSA group $(\mathrm{p}=0.148)$. Cumulative incidences of the primary end-point are shown in figure 2 . There were no significant differences in the rates of individual components of the composite end-points in the PCI and CABG subgroups (supplementary table E4).

In CPAP-treated sleepy OSA patients the unadjusted and adjusted hazard ratio (HR) values for MACCEs were 1.51 (95\% CI 0.86-2.67; p=0.151) and 0.96 (95\% CI 0.40-2.31; p=0.923), respectively. Age (HR 1.04, 95\% CI 1.00-1.08; $\mathrm{p}=0.056$ ) and previous revascularisation (HR 1.90, 95\% CI 1.01-3.55; $\mathrm{p}=0.045$ ) were associated with increased risk for MACCEs, whereas CABG at baseline (HR 0.39, 95\% CI 0.15-0.99; $\mathrm{p}=0.045$ ) reduced the risk for MACCEs (table 3). Excluding 37 nonadherent OSA patients from the analysis resulted in a nonsignificant increase in the adjusted HR value for MACCEs (1.21, 95\% CI 0.45$3.22 ; \mathrm{p}=0.704$ ) for treated OSA compared with patients with no OSA (supplementary table E5). On-treatment analysis of CPAP usage of at least $4 \mathrm{~h}$ per night within the OSA group showed an adjusted HR of 1.18 (95\% CI 0.36-3.86; $\mathrm{p}=0.781$ ).

\section{Excessive daytime sleepiness}

ESS scores decreased significantly from $12.2 \pm 2.5$ at baseline to $9.6 \pm 3.7$ at 3-month follow-up, to $9.3 \pm 3.1$ at 1 -year follow-up and to $8.5 \pm 3.4$ at 2 -year follow-up (all $\mathrm{p}<0.001$ versus baseline).

\section{Adverse events}

No serious adverse events related to CPAP were observed in the OSA group. Patient-reported side-effects during CPAP were consistent with the known tolerability profile of CPAP, and included dry mouth, nasal symptoms, claustrophobia, insomnia, noise problems and mask fit. 


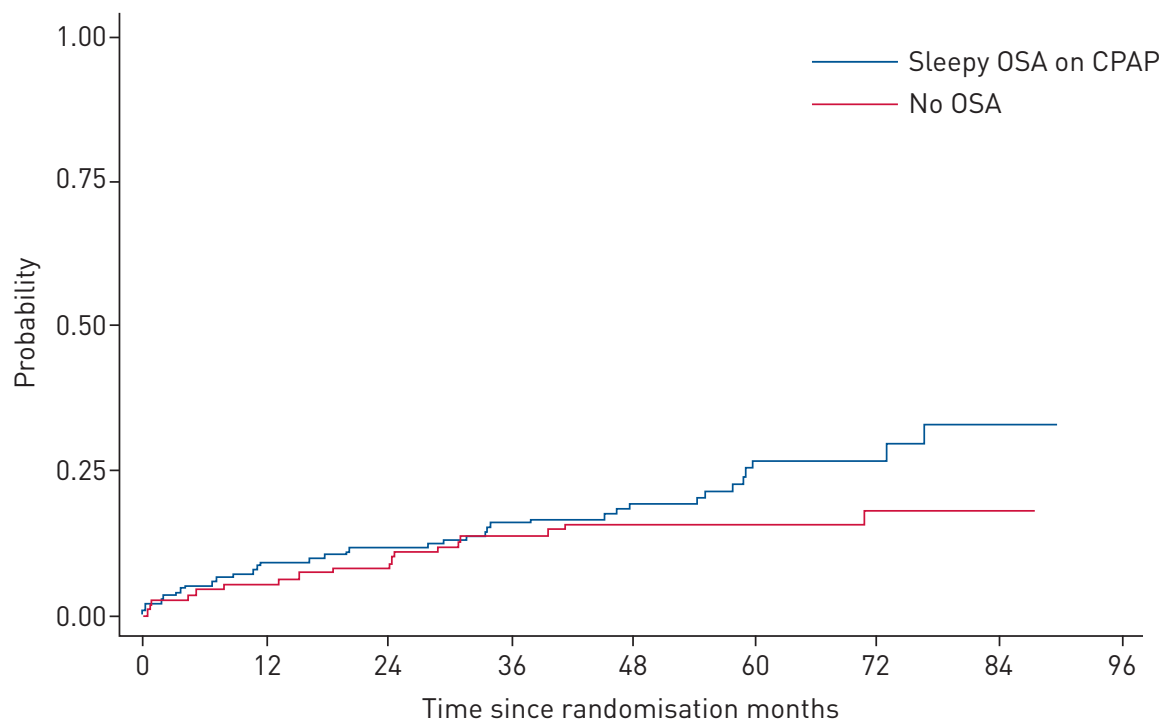

At risk $n$

$\begin{array}{llllcccccc}\text { Sleepy OSA } & 155 & 139 & 134 & 116 & 89 & 53 & 31 & 7 & 0 \\ \text { No OSA } & 112 & 105 & 102 & 88 & 67 & 47 & 31 & 13 & 0\end{array}$

FIGURE 2 Cumulative incidences of the composite end-point in the study population. OSA: obstructive sleep apnoea; CPAP: continuous positive airway pressure.

\section{Discussion}

To the best of our knowledge, this is the first prospective, observational study comparing risk for MACCEs in a revascularised CAD cohort with sleepy OSA receiving CPAP treatment with risk in patients without OSA. The results showed that the risk for MACCEs in revascularised CAD patients with sleepy OSA on CPAP was similar to that in CAD patients without OSA. In a multivariate analysis, age and former revascularisation were associated with an increased risk for MACCEs, while CABG appeared to be superior to PCI for reducing the risk for subsequent MACCEs in this cohort.

TABLE 3 Cox regression analysis of baseline covariables associated with risk for adverse cardiovascular outcomes in revascularised patients with coronary artery disease and sleepy obstructive sleep apnoea (OSA) treated with continuous positive airway pressure (CPAP) versus no OSA

\begin{tabular}{|c|c|c|c|c|}
\hline & \multicolumn{2}{|l|}{ Univariate } & \multicolumn{2}{|l|}{ Multivariate } \\
\hline & Hazard ratio $(95 \% \mathrm{CI})$ & p-value & Hazard ratio $(95 \% \mathrm{CI})$ & p-value \\
\hline Sleepy OSA on CPAP versus no OSA & $1.51(0.86-2.67)$ & 0.151 & $0.96(0.40-2.31)$ & 0.923 \\
\hline Age & $1.04(1.00-1.07)$ & 0.027 & $1.04(1.00-1.08)$ & 0.056 \\
\hline Female versus male & $0.62(0.26-1.44)$ & 0.262 & $0.62(0.26-1.50)$ & 0.292 \\
\hline Apnoea-hypopnoea index & $1.01(1.00-1.02)$ & 0.072 & $1.01(0.99-1.04)$ & 0.220 \\
\hline BMI & $1.02(0.97-1.08)$ & 0.417 & $1.00(0.93-1.08)$ & 0.969 \\
\hline CABG versus $\mathrm{PCI}$ & $0.39(0.17-0.91)$ & 0.029 & $0.39(0.15-0.99)$ & 0.048 \\
\hline Current smoking & $1.04(0.54-2.03)$ & 0.899 & $1.28(0.62-2.64)$ & 0.505 \\
\hline Hypertension & $1.54(0.89-2.68)$ & 0.126 & $1.38(0.77-2.48)$ & 0.283 \\
\hline Diabetes mellitus & $1.30(0.69-2.42)$ & 0.416 & $1.10(0.55-2.19)$ & 0.782 \\
\hline Acute MI & $1.15(0.68-1.97)$ & 0.600 & $1.15(0.61-2.18)$ & 0.667 \\
\hline Previous $\mathrm{PCl}$ or CABG & $2.13(1.20-3.79)$ & 0.010 & $1.90(1.01-3.55)$ & 0.045 \\
\hline Pulmonary disease & $1.06(0.45-2.47)$ & 0.901 & $0.92(0.38-2.23)$ & 0.860 \\
\hline LVEF & $0.99(0.95-1.02)$ & 0.384 & $1.00(0.97-1.03)$ & 0.889 \\
\hline
\end{tabular}

BMI: body mass index; CABG: coronary artery bypass grafting; $\mathrm{PCl}$ : percutaneous coronary intervention; MI: myocardial infarction; LVEF: left ventricular ejection fraction. ${ }^{\#}: n=267 ; 54$ patients reached the composite end-point. 
Many CAD patients experience MACCEs in the years after intervention, despite advances in medical treatment and revascularisation techniques [2,3], and it has been suggested that OSA may play an important role in this context. YUmino et al. [4] documented MACCEs after PCI in almost $24 \%$ of patients with concomitant OSA compared with $5 \%$ of those without OSA over a 6-month follow-up. More recently, a single-centre study of 340 consecutive CAD patients treated with a drug-eluting stent reported that the incidence of a major cardiac event (MACE; new revascularisation, myocardial infarction or cardiac mortality) over a median 2 -year follow-up was $25 \%$ in patients with OSA (AHI $\geqslant 15$ events $\cdot \mathrm{h}^{-1}$ on CRPG) compared with $16 \%$ in those with AHI $<15$ events $\cdot \mathrm{h}^{-1}(\mathrm{p}=0.038)$ and that OSA was an independent predictor of MACEs, nearly doubling the risk [5]. In a much larger cohort of CAD patients undergoing PCI [6], LeE et al. [6] reported that $45 \%$ of 1311 patients had OSA (AHI $\geqslant 15$ events $\mathrm{h}^{-1}$ on CRPG within 7 days after revascularisation). In this multicentre study conducted in five countries, OSA was a predictor of MACCEs over a median follow-up of almost 2 years, with an adjusted HR of 1.6 independent of age, sex, ethnicity, BMI, diabetes mellitus and hypertension. Moreover, in a smaller study of 67 CAD patients undergoing CABG, MACCEs were observed in $35 \%$ of OSA patients (AHI $\geqslant 15$ events $\cdot \mathrm{h}^{-1}$ on CRPG) compared with $16 \%$ in patients with $\mathrm{AHI}<15$ events $^{-1} \mathrm{~h}^{-1}(\mathrm{p}=0.02)$ during a mean follow-up of 4.5 years and OSA was an independent predictor of incident MACCEs with a 4 -fold risk increase in multivariate analysis [7].

Less is known about the impact of CPAP treatment on long-term cardiovascular outcomes in CAD patients with OSA. A retrospective analysis of 55 CAD patients with OSA over a mean follow-up of 7.3 years showed a significantly lower occurrence of the composite end-point (cardiovascular death, acute coronary syndrome, hospitalisation for cardiac failure or need for revascularisation) in those compliant with prescribed CPAP therapy [10]. In another retrospective study of 371 revascularised CAD patients with OSA, CASSAR et al. [12] found that the cardiac death rate over 5 years after PCI was significantly lower in 175 patients treated with CPAP (3\% versus 10\% in 196 untreated patients). In a later prospective follow-up study of 186 post-myocardial infarction patients, treated OSA patients had a lower risk for recurrent myocardial infarction (adjusted $\mathrm{HR}$ 0.16) and revascularisation (adjusted HR 0.15) than untreated OSA patients [17]. Until recently, there was a lack of prospective studies regarding the risk for incident MACCEs in revascularised CAD patients with symptomatic OSA patients on CPAP treatment, partly because it is unethical to randomise such patients to no treatment. However, because many cardiac patients do not report daytime symptoms, it has been argued that RCTs are crucial to address whether such patients should be offered CPAP treatment to reduce MACCEs [18].

In this context, the RICCADSA trial was the first RCT to investigate the impact of CPAP on MACCEs in revascularised $\mathrm{CAD}$ patients [13]. The study demonstrated that routine CPAP prescription to CAD patients with nonsleepy OSA did not significantly reduce long-term adverse outcomes in the ITT population, but that risk reduction was observed after adjustment for baseline comorbidities and CPAP adherence [13]. The most recent study investigating the secondary prevention potential of CPAP in patients with OSA is the SAVE (Sleep Apnoea Cardiovascular End-points) trial. In this large, multicentre, multinational cohort study, 2717 patients with moderate-to-severe OSA and established cardiovascular disease were assigned to CPAP treatment plus usual care $(n=1359)$ or usual care $(n=1358)$ [14]. After a mean follow-up of 3.7 years, the results showed that CPAP treatment did not prevent cardiovascular events in the ITT population despite a significant reduction in daytime sleepiness and improvement in health-related quality of life and mood [14]. Half of the SAVE study population had established CAD (of whom $77 \%$ had a history of revascularisation), and $20 \%$ of the study population had an ESS score between 11 and 15, indicating mild to moderate daytime sleepiness. In the CPAP group, mean device usage was $3.3 \mathrm{~h}$ per night and $42 \%$ had good adherence (CPAP usage $\geqslant 4 \mathrm{~h}$ per night). The results of a secondary analysis suggested that patients who were adherent to CPAP had a lower risk for stroke (unadjusted HR 0.56; $\mathrm{p}=0.05$ ). The SAVE study results indicate that getting nonsleepy patients to comply with CPAP is challenging, which is consistent with the results of the RCT arm of the RICCADSA cohort. Moreover, on-treatment analysis of the RCT arm of the RICCADSA cohort as well as propensity score-matched secondary analysis of the SAVE data suggest that CPAP adherence of at least $4 \mathrm{~h}$ per night is required to achieve cardiovascular benefits. In the observational arm of the RICCADSA cohort, $24 \%$ of sleepy OSA patients who started CPAP at baseline returned the device within 2 years, which is comparable with the adherence rates in sleep clinic cohorts with CAD [19]. Thus, CPAP treatment is feasible in CAD populations with sleepy OSA, significantly reduces daytime sleepiness and may also reduce long-term cardiovascular risk. The current analysis did not identify a dose-response relationship for CPAP usage and the primary end-point in sleepy OSA patients, probably due to the small absolute number of patients in each CPAP usage category. Interestingly, secondary analysis excluding 37 nonadherent OSA patients at 2-year follow-up resulted in a nonsignificant increase in the hazard ratio for OSA patients on CPAP versus no OSA, most likely due to the proportional increase in comorbidity in the OSA group when the less severe patients were eliminated. Moreover, there was a significant increase in the degree of obesity over 
time in patients with OSA adherent to CPAP. Such changes, in addition to other uncontrolled lifestyle factors and possible improvements in OSA severity over time associated with better control of underlying $\mathrm{CAD}$, at least in some patients, might influence the long-term impact of CPAP treatment in CAD patients with concomitant OSA.

The strengths of this study include its prospective design for CPAP-treated patients with CAD and sleepy OSA compared with no OSA, with only three patients lost to follow-up. Although the inclusion rate for eligible patients for sleep screening for the whole RICCADSA trial was 53\%, the inclusion design was consecutive and there were no significant differences in baseline characteristics of patients undergoing versus not undergoing sleep study [20].

The current study also had a number of limitations. This was a single-centre trial with two sites, meaning that the results are not generalisable across geographic regions. Moreover, "sleepy" OSA was defined based on an ESS score threshold, which may not reflect objective sleepiness. However, the ESS is a generally accepted tool for subjective daytime sleepiness and other methods (e.g. the objective Multiple Sleep Latency Test [21]) were not feasible for the large-scale cardiac population. Furthermore, the trial had an open-label design and there was no placebo control arm for the OSA group. However, there is no true sham CPAP or other appropriate placebo for CPAP in a long-term trial in CAD patients and this is also inappropriate for ethical reasons. Finally, results of the observational studies must be interpreted with care because CPAP usage is mainly patient-driven and self-selection bias cannot be excluded. This kind of bias can only be avoided by an ITT analysis of a randomised trial. Lack of a control group of untreated OSA in this observational arm is therefore an additional limitation. However, a randomisation of sleepy CAD patients to control would not be ethically feasible.

It should be emphasised that the original sample size calculation for the RICCADSA trial was performed for the RCT arm and was intended to provide adequate power to detect a statistically significant difference between treatment groups based on the event rate assumptions used. Separate sample size and power estimates were not performed for the observational arm. In the absence of a control group of untreated sleepy OSA patients, we chose the no OSA patient group to act as controls to show that sleepy OSA patients treated with CPAP would not be inferior to patients without OSA in this CAD population. The multivariate analysis showed a HR of 0.96 (95\% CI 0.40-2.31). To better prove noninferiority (i.e. to reach a CI <1.2), the observational arm study would therefore have needed 922 patients ( 387 no OSA and 535 sleepy OSA on CPAP) using the same event and dropout rates.

In conclusion, while age and previous revascularisation were associated with increased risk for MACCEs and $\mathrm{CABG}$ was protective compared with PCI regarding long-term adverse outcomes, the risk for MACCEs in this revascularised CAD cohort with sleepy OSA treated with CPAP was similar to that in CAD patients without OSA. The potential role of CPAP in secondary cardiovascular prevention protocols for patients with CAD needs to be further evaluated in larger clinical cohorts.

\section{Acknowledgements}

The authors gratefully acknowledge the independent clinical event committee, Lennart Welin (Sahlgrenska Academy, University of Gothenburg, Sweden), the data monitoring board, Marina Modin (Skaraborg Hospital, Skövde, Sweden), and thank sleep technologist Paul Murphy (Sleep Medicine Institute, Gothenburg, Sweden) for scoring of the in-hospital PSG recordings and research secretary Eija Magnusson (Skaraborg Hospital, Skövde, Sweden) for her professional assistance in data entry and coordinating quality control of patient records and data follow-up during the whole trial period. Editing assistance was provided by Nicola Ryan (independent medical writer; Auckland, New Zealand) funded by ResMed.

Author Contributions: Y. Peker designed the study in 2005. Y. Peker, E. Thunström and H. Glantz performed the patient recruitment and clinical follow-ups. Y. Peker, K. Wegscheider and C. Eulenburg performed the statistical analysis. All authors interpreted the data, prepared the manuscript and drafted the article. Y. Peker obtained study funding and takes full responsibility for the work as a whole, including the study design, access to data, and the decision to submit and publish the manuscript. All authors approved the manuscript in its final form.

\section{References}

1 Peker Y, Franklin K, Hedner J. Coronary artery disease and sleep apnea. In: Kryger MH, Roth TT, Dement WT, eds. Principals and Practice of Sleep Medicine. 6th Edn. Philadelphia, Elsevier, 2017; pp. 1264-1270.

2 Serruys PW, Morice MC, Kappetein AP, et al. Percutaneous coronary intervention versus coronary-artery bypass grafting for severe coronary artery disease. N Engl J Med 2009; 360: 961-972.

3 Kappetein AP, Feldman TE, Mack MJ, et al. Comparison of coronary bypass surgery with drug-eluting stenting for the treatment of left main and/or three-vessel disease: 3-year follow-up of the SYNTAX trial. Eur Heart J 2011; 32: 2125-2134.

4 Yumino D, Tsurumi Y, Takagi A, et al. Impact of obstructive sleep apnea on clinical and angiographic outcomes following percutaneous coronary intervention in patients with acute coronary syndrome. Am J Cardiol 2007; 99: 26-30. 
5 Zhang JJ, Gao XF, Ge Z, et al. Obstructive sleep apnea affects the clinical outcomes of patients undergoing percutaneous coronary intervention. Patient Prefer Adherence 2016; 10: 871-878.

6 Lee $\mathrm{CH}$, Sethi R, Li R, et al. Obstructive sleep apnea and cardiovascular events after percutaneous coronary intervention. Circulation 2016; 133: 2008-2017.

7 Uchôa CH, Danzi-Soares NJ, Nunes FS, et al. Impact of OSA on cardiovascular events after coronary artery bypass surgery. Chest 2015; 147: 1352-1360.

8 Engleman HM, Martin SE, Kingshott RN, et al. Randomised placebo controlled trial of daytime function after continuous positive airway pressure (CPAP) therapy for the sleep apnoea/hypopnoea syndrome. Thorax 1998; 53 : 341-345.

9 Epstein LJ, Kristo D, Strollo PJ Jr, et al. Clinical guideline for the evaluation, management and long-term care of obstructive sleep apnea in adults. J Clin Sleep Med 2009; 5: 263-276.

10 Milleron O, Pilliére R, Foucher A, et al. Benefits of obstructive sleep apnoea treatment in coronary artery disease: a long-term follow-up study. Eur Heart J 2004; 25: 728-734.

11 Doherty LS, Kiely JL, Swan V, et al. Long-term effects of nasal continuous positive airway pressure therapy on cardiovascular outcomes in sleep apnea syndrome. Chest 2005; 127: 2076-2084.

12 Cassar A, Morgenthaler TI, Lennon RJ, et al. Treatment of obstructive sleep apnea is associated with decreased cardiac death after percutaneous coronary intervention. J Am Coll Cardiol 2007; 50: 1310-1314.

13 Peker Y, Glantz H, Eulenburg C, et al. Effect of positive airway pressure on cardiovascular outcomes in coronary artery disease patients with nonsleepy obstructive sleep apnea. The RICCADSA randomized controlled trial. Am J Respir Crit Care Med 2016; 194: 613-620.

14 McEvoy RD, Antic NA, Heeley E, et al. CPAP for prevention of cardiovascular events in obstructive sleep apnea. N Engl J Med 2016; 375: 919-931.

15 Peker Y, Glantz H, Thunström E, et al. Rationale and design of the randomized intervention with CPAP in coronary artery disease and sleep apnoea - RICCADSA trial. Scand Cardiovasc J 2009; 43: 24-31.

16 Fisher LD, Lin DY. Time-dependent covariates in the Cox proportional-hazards regression model. Annu Rev Public Health 1999; 20: 145-157.

17 Garcia-Rio F, Alonso-Fernández A, Armada E, et al. CPAP effect on recurrent episodes in patients with sleep apnea and myocardial infarction. Int J Cardiol 2013; 168: 1328-1335.

18 Somers VK, White DP, Amin R, et al. Sleep apnea and cardiovascular disease. An American Heart Association/ American College of Cardiology Foundation Scientific Statement from the American Heart Association Council for High Blood Pressure Research Professional Education Committee, Council on Clinical Cardiology, Stroke Council, and Council on Cardiovascular Nursing Council. Circulation 2008; 118: 1080-1111.

19 Sampol G, Rodés G, Romero O, et al. Adherence to nCPAP in patients with coronary disease and sleep apnea without sleepiness. Respir Med 2007; 101: 461-466.

20 Glantz H, Thunström E, Herlitz J, et al. Occurrence and predictors of obstructive sleep apnea in a revascularized coronary artery disease cohort. Ann Am Thorac Soc 2013; 4: 350-356.

21 Wise MS. Objective measures of sleepiness and wakefulness: application to the real world? J Clin Neurophysiol 2006; 23: 39-49. 